\title{
Collimation effects on large CPA compressors
}

\author{
R. Heathcote $\cdot$ M. Galimberti $\cdot$ R. J. Clarke $\cdot$ \\ T. B. Winstone $\cdot$ I. O. Musgrave $\cdot$ C. Hernandez-Gomez
}

Received: 31 May 2013/Accepted: 7 January 2014/Published online: 8 February 2014

(C) The Author(s) 2014. This article is published with open access at Springerlink.com

\begin{abstract}
Modelling and analysis have been performed to show a link between laser collimation tolerances and dispersion effects in chirped pulse amplification (CPA) compressors. These show that an un-collimated beam can present itself as residual dispersion in a CPA system which uncorrected will have adverse effects on laser power and intensity. The techniques of shearing interferometry and beam propagation over large distances for setting beam collimation have been assessed for their limitations in measuring wavefront radii. An analysis of the sensitivity of CPA systems to non-collimated beams has been studied. This effect has been practically demonstrated for a highpower Nd:glass laser and the best techniques for alignment are discussed.
\end{abstract}

\section{Introduction}

The technique of chirped pulsed amplification (CPA) has been established for many years as a means of producing the highest peak power lasers [1]. A CPA system consists of a stretcher that introduces wavelength-dependent delays to the spectral components of a short pulse (typically $<1 \mathrm{ps})$, to produce a long pulse $(\sim 1-3 \mathrm{~ns})$ for the purposes of amplification to higher energy, avoiding non-linear optical effects due to intensity. This amplified beam is then re-compressed to produce a short high-power laser pulse. The compressor in most schemes consists of a series

R. Heathcote $(\square) \cdot$ M. Galimberti · R. J. Clarke ·

T. B. Winstone - I. O. Musgrave · C. Hernandez-Gomez Central Laser Facility, Science and Technology Facilities

Council, Rutherford Appleton Laboratory, Harwell Oxford,

Didcot, Oxfordshire, UK

e-mail: robert.heathcote@stfc.ac.uk of diffraction gratings, which restores the short pulse by reversing the delays introduced in the stretcher.

In high-power Nd:glass lasers, such as the Vulcan laser system at the Rutherford Appleton Laboratory [2], the low bandwidth and large beam sizes (beam diameters $\sim 10-60 \mathrm{~cm})$ are best matched to a single pass compressor configuration. This design allows for maximum compressed pulse energies at the marginal expense of increased pulse duration and lateral dispersion [3].

It has been known for many years that beam collimation has a role in the maximum achievable pulse compression [4] but to what degree has been less well established.

In this paper, we present models of the two Vulcan single pass compressor CPA beamlines to demonstrate the effect of collimation errors on compressed pulse duration.

\section{Analysis}

The beam divergence has an effect on the focusability of the beam due to the nature of the single pass compressor. In single pass the bandwidth $\Delta \lambda=\lambda_{\max }-\lambda_{\min }$ is laterally sheared by the gratings (Fig. 1) and for a divergent beam effectively creates multiple source points which are reimaged by the focusing optics to a line (Fig. 2b). To first order, the horizontal size of the focal spot is determined by the size of the shear $S$, the source distance $R$ (i.e. the radius of curvature of the wavefront) and the focal length $f$ of the focusing optics. Taking into account the diffraction limit, the spot size will only be affected when

$|R| \lesssim \frac{S D}{1.22 \lambda}$

for a beam of diameter $D . S$ is dependent on the bandwidth and the dispersion of the grating. 


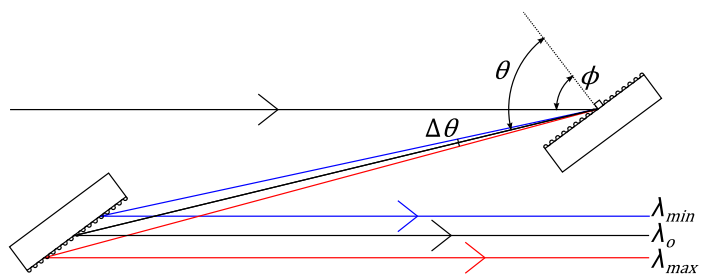

Fig. 1 Spectral shearing in a single pass compressor

(a)

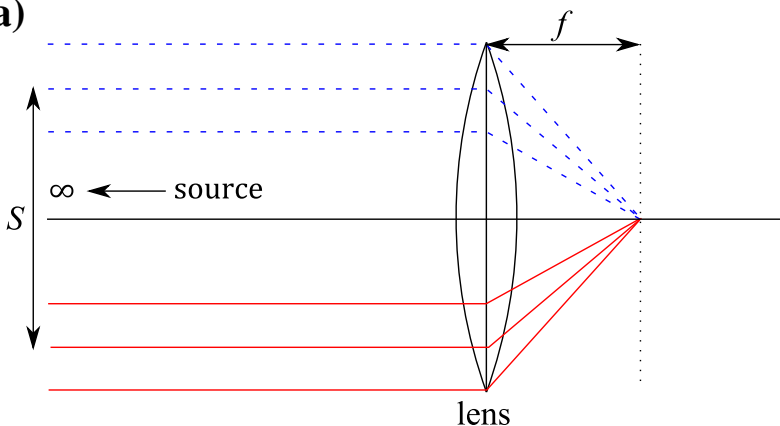

(b)

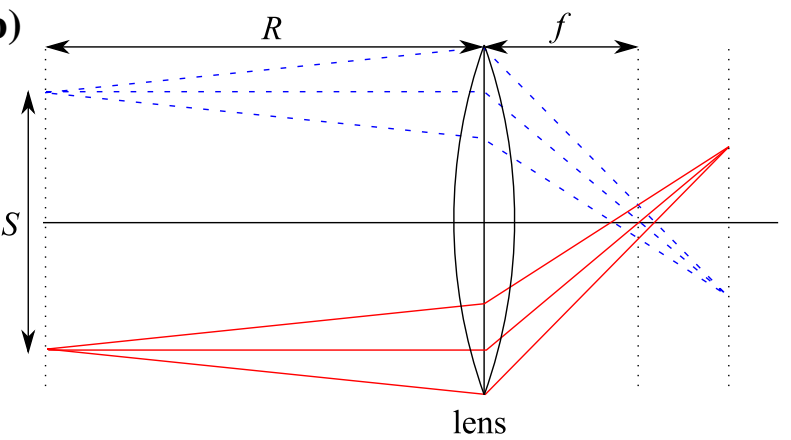

Fig. 2 A laterally sheared beam will image to the a single spot when collimated b line when divergent

The grating equation for order $m$ is given by

$\frac{m \lambda}{d}=(\sin \phi+\sin \theta)$

where $\phi$ and $\theta$ are shown in Fig. 1 and $d$ is the diffraction grating ruling separation.

The angular dispersion of the bandwidth $\Delta \theta=\theta_{\lambda_{\max }}$ $-\theta_{\lambda_{\min }}$ leads to a shear $S$ given by Eq. (3).

$S=z_{o} \cos \phi\left[\frac{\sin \left(\theta_{\lambda_{\max }}-\theta_{o}\right)}{\sin \left(\frac{\pi}{2}-\theta_{\lambda_{\max }}\right)}+\frac{\sin \left(\theta_{o}-\theta_{\lambda_{\min }}\right)}{\sin \left(\frac{\pi}{2}+\theta_{\lambda_{\min }}\right)}\right]$

where $z_{o}$ is the grating separation and $\theta_{o}$ the diffracted angle of the centre wavelength $\lambda_{o}$.

For example, for the parameters of the beamlines detailed in Table 1 give values for the shears of 74 and $208 \mathrm{~mm}$ for the $100 \mathrm{TW}$ and Petawatt beamlines, respectively. From Eq. (1), these correspond to a lower limit on the radius of wavefront curvatures of $\sim 11$ and $\sim 97 \mathrm{~km}$.
Table 1 Compressor specifications

\begin{tabular}{lll}
\hline Beamline & $100 \mathrm{TW}$ & Petawatt \\
\hline Beam diameter $D$ & $200 \mathrm{~mm}$ & $600 \mathrm{~mm}$ \\
Grating line density $\frac{1}{d}$ & $1,740 \mathrm{~mm}^{-1}$ & $1,490 \mathrm{~mm}^{-1}$ \\
Angle of incidence $\phi$ & $61^{\circ}$ & $48^{\circ}$ \\
Centre wavelength $\lambda_{0}$ & $1,053.5 \mathrm{~nm}$ & $1,055.5 \mathrm{~nm}$ \\
Bandwidth $\Delta \lambda$ & $2 \mathrm{~nm}$ & $5 \mathrm{~nm}$ \\
Grating separation $z_{0}$ & $3.5 \mathrm{~m}$ & $13 \mathrm{~m}$ \\
Stretching factor & $458 \mathrm{ps} \mathrm{nm}^{-1}$ & $326 \mathrm{ps} \mathrm{nm}^{-1}$ \\
\hline
\end{tabular}

\section{Modelling}

Modelling was performed using the commercial Zemax ${ }^{\circledR}$ ray-tracing software [5] to study the effects of un-collimated beams in CPA compressors in comparison with a grating parallelism error. Models were produced for the Vulcan Petawatt beamline compressor which consists of a pair of 940-mm diameter gold gratings (Fig. 3) and the Vulcan 100 TW beamline compressor which have $420 \times$ $210 \mathrm{~mm}$ gold gratings. Table 1 gives the relevant parameters of the compressors.

The bandwidth was modelled with wavelength samples at $0.5 \mathrm{~nm}$ intervals with a triangular intensity function varying from 0.5 at the extremes to 1 in the centre. Both systems used models of the actual $\mathrm{f} / 3$ off-axis parabola focusing optics. The models were set with the source distance variable to simulate wavefront radii. In reality, beam collimation is determined by the final beam telescopes.

The FWHM of the focal spot in the dispersion plane as a function of $R$ was modelled for the Petawatt beamline and the 100 TW beamline (Fig. 4) and broadly agrees with the lower limit of $R$ of $\sim 97$ and $\sim 11 \mathrm{~km}$, respectively, from Eq. (1) based on the shear created by the spectral bandwidth of the beams.

Modelling was also performed to illustrate the effect of the misalignment of the gratings. Figure $5 \mathrm{a}$ and $\mathrm{b}$ show that at focus a divergent beam with a wavefront radius of curvature of $25 \mathrm{~km}$ has an identical focal spot to that formed by a perfectly collimated beam in a misaligned compressor with an angle of $0.5 \mathrm{mrad}$ between the surfaces in the horizontal plane. Note, the image plane of Fig. 5a is offset from the focal plane of the parabola and image plane of Fig. $5 b$ by $135 \mu \mathrm{m}$. These two effects can be combined so that the dispersion created by the divergence is compensated for by the opposite dispersion introduced by rotating the second grating (Fig. 5c). This will form a diffraction limited focal spot at the $135 \mu \mathrm{m}$ offset from the focal plane of the parabola, but will introduce angular chirp, thus increasing the pulse duration and reducing the intensity of the focal spot [6].

This effect on the pulse-length has been simulated using a ray-tracing code developed in-house that simulated the phase 
(a)

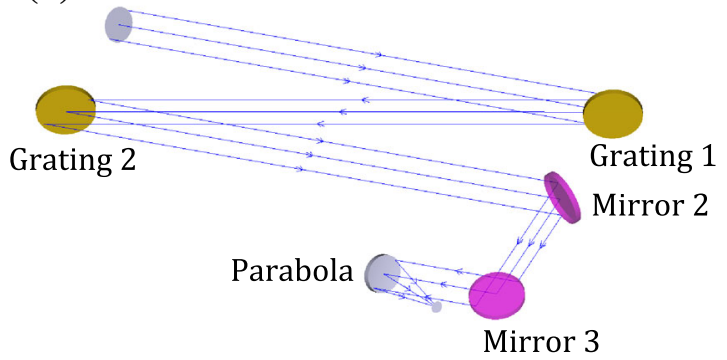

(b)

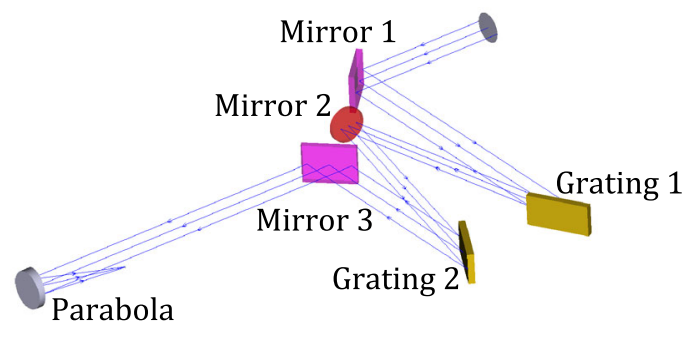

Fig. 3 Zemax models of the a Petawatt beamline and b 100 TW beamline compressors

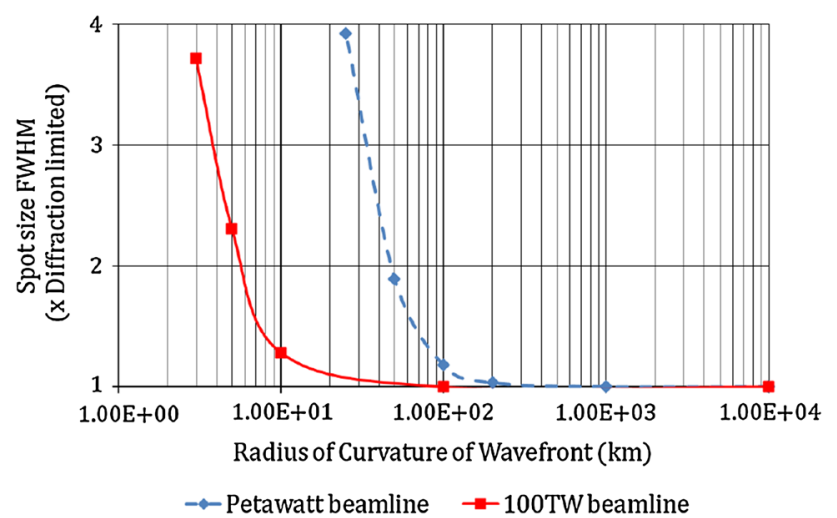

Fig. 4 Spot FWHM as a function of radius of curvature of wavefront
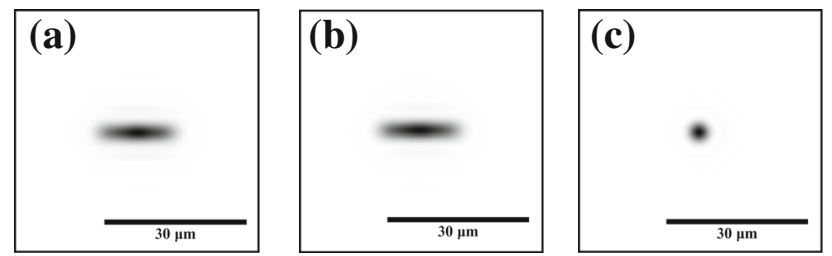

Fig. 5 Zemax simulation of the Petawatt beamline focal spot (point spread function using the fast fourier transform method-PSF FFT) with a a radius of curvature of $25 \mathrm{~km}, \mathbf{b}$ a collimated beam and $0.5 \mathrm{mrad}$ rotation of the second grating and $\mathbf{c}$ a radius of curvature of $25 \mathrm{~km}$ compensated with a $0.5 \mathrm{mrad}$ rotation of the second grating

of a sample of constituent wavelengths for a CPA pulse and calculated the temporal and spatial profile of the beam. Figure 6 compares the ideal configuration of parallel gratings and a collimated beam with a divergent beam compensated with grating rotation. It can be seen in Fig. $6 \mathrm{~d}$ that even though the focal spot is roughly maintained, the tilted intensity front in Fig. 6b has increased the pulse-length from 391 fs (Fig. 6c) to 1.7 ps (Fig. 6d). This method of compensating divergence by grating rotation is clearly not a suitable technique due to its strong effect in the temporal domain.

In practice, a temporally elongated pulse may be due to a number of factors that influence pulse compression but as illustrated it is important that the collimation of the beam is also considered.

\section{Methods for determining collimation}

Commercial systems such as Shack-Hartmann wavefront sensors are available for measuring wavefront quality at small aperture $(\sim 5 \mathrm{~mm})$ but in practice, implementing these for large diameter beams requires imaging systems that are fully characterised which become increasingly difficult and cost prohibitive the larger the aperture. Traditional methods that have been adopted on the Vulcan laser system at the Rutherford Appleton Laboratory for beam collimation have been shearing interferometry and measuring the divergence of two objects in the beam propagated over a large $(\sim 50 \mathrm{~m})$ distance.

A lateral shearing interferometer [7] uses a slightly wedged high-quality optical flat known as a shear plate. The shear plate is used at a $45^{\circ}$ angle to the beam and the front and rear reflections interfere in the region of overlap. The fringe pattern orientation gives an indication of the collimation. When the beam is said to be collimated the fringe pattern is parallel with the intersection axis of the surface planes of the wedge. The accuracy of this method is determined by the ability to measure the fringe orientation with respect to the reference line. This is dependent on the fringe separation, width of beam, shear distance and residual beam aberrations. A shear and fringe separation of $1 / 5$ of the diameter of the beam and accuracy of $\pm 5^{\circ}$ fringe angle gives wavefront radius of curvature of $|R| \gtrsim D^{2} / 2 \lambda$. For a beam diameter of $100 \mathrm{~mm}$ and $\lambda=1,053 \mathrm{~nm}$, this corresponds to a radius of curvature $>5 \mathrm{~km}$ which is a wavefront error of $\sim \lambda / 4$. To maintain this accuracy, it requires shear plates large enough for the beam diameter. Sub-sampling a $200 \mathrm{~mm}$ beam at $100 \mathrm{~mm}$ will produce an accuracy to a radius of $5 \mathrm{~km}$ but a wavefront error of $\lambda$.

An alternative method for determining collimation is to place two objects in the beam, and measure the separation of their shadows after the beam has propagated over a large distance. The radius of curvature of the wavefront can be obtained from the separation of the objects $D$; in most cases, thin wires, the propagation distance $x$ and the change in the separation of the objects' diffracted shadows, $\delta D$, which are related by $R=D x / \delta D$. The limit on the accuracy 
Fig. 6 Temporal beam profiles of the Petawatt beamline in the dispersive plane in the near field $(\mathbf{a}, \mathbf{b})$ and far field (c, d) after compression in an ideal configuration (a, c) and compensated divergence with grating rotation $(\mathbf{b}, \mathbf{d})$ (a)

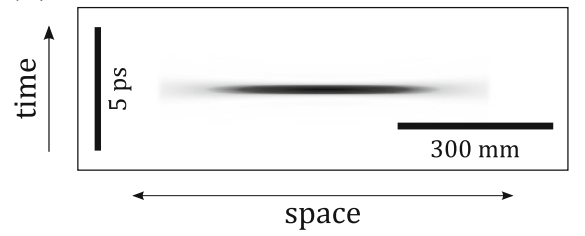

(c)

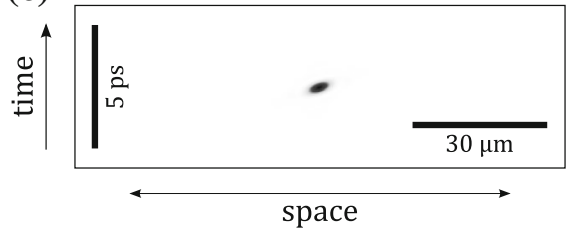

(b)

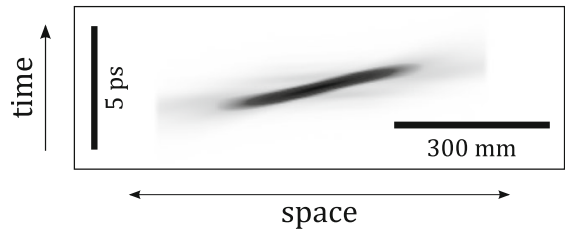

(d)

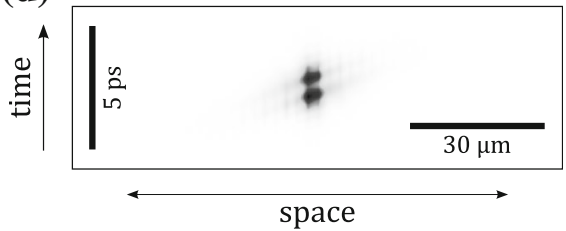

of this method depends primarily on the ability to propagate a sufficiently large distance and the error associated with measuring the separation of the diffraction patterns. For use on the Petawatt beamline, the limits are $x=30 \mathrm{~m}$, $D=0.5 \mathrm{~m}$ and the ability to measure the separation $\delta D=$ to $\pm 1 \mathrm{~mm}$ which gives a $|R|$ of $\geq 15 \mathrm{~km}$.

These methods for measuring $R$ assume that there is access to the beam. However, for high-power short pulse lasers it is often necessary to propagate the beam under vacuum. This can affect collimation as the effective focal lengths of lenses are different in air and vacuum. With the optical system under vacuum, it makes standard collimation measurements mechanically impossible; so, re-collimation has to be done at atmosphere and then adjusted based on calculation. For example, the final telescope for the Petawatt beamline is a $3 \times$ expander for which the separation of the lenses has to change by $\sim 14 \mathrm{~mm}$ between air and vacuum.

The measurement can be also be complicated by imperfections in the flatness of the optical surfaces which can change the beam collimation as it passes along the optical chain. These errors are not addressed here.

As shown in Sect. 1, the accuracy of collimation required by a CPA beam can be greater than is achievable with the techniques described. In the next section, we present a more suitable method for collimation in combination with compressor alignment.

\section{Compressor alignment observations and techniques}

Optimum pulse compression requires that the CPA gratings be parallel in both surface and groove orientation to avoid introducing temporal aberrations.

The approach to fine tuning the alignment is to use a dichroic laser and to double pass the compressor by retro reflecting the beam. In double passing the compressor, the sensitivity of grating alignment is increased whilst also making the process insensitive to collimation errors, as the spectral shear created in a single pass will be undone on the return path. This is also true of laser systems that utilise double pass and four grating compressors. The diagnosis of the alignment is done with the far-field focal spot of the retro-reflected beam after the return pass of the compressor. To first order, a horizontal offset between foci of the two component wavelengths indicates a grating surface parallelism error, and a vertical offset indicates a groove parallelism error. By moving the second grating to overlap the spots, these errors can be removed. The horizontal offset is corrected by horizontally rotating the grating and maintaining the far-field position with the retro reflecting mirror. The vertical offset is corrected by rolling the grating and compensating the far-field movement by vertically rotating the grating.

Overlapping the spots in double pass will establish that in single pass no residual dispersion is attributable to grating misalignment. The angular sensitivity $\varphi$ of this method is dependent on the accuracy to which a spot

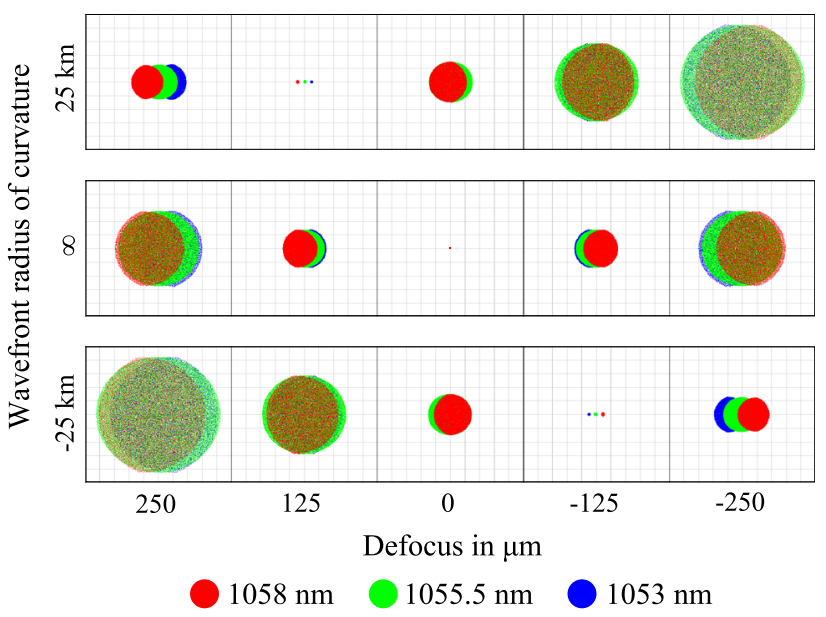

Fig. 7 Through focus spot diagram for differing wavefront radius of curvature. The position of the tightest focus for the divergent and convergent case is offset from the ideal focal plane of the parabola. In these cases, the defocus is not symmetric about the tightest focal spot 
Fig. 8 Through focus spot before correction (a) as measured on the Vulcan Petawatt beamline (b). Zemax simulated focal spot (PSF FFT) with alignment laser at 1,053 and $1,055 \mathrm{~nm}$. This corresponds to a converging beam with wavefront radius of $\sim 35 \mathrm{~km}$ (a)

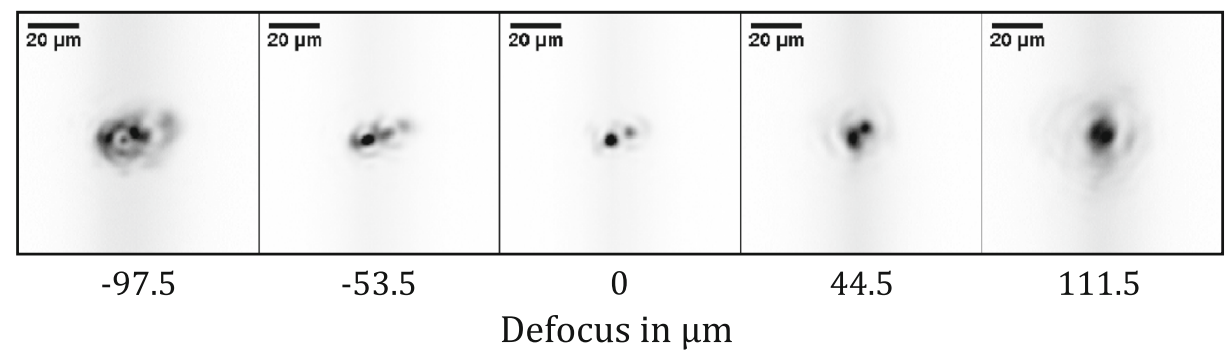

(b)

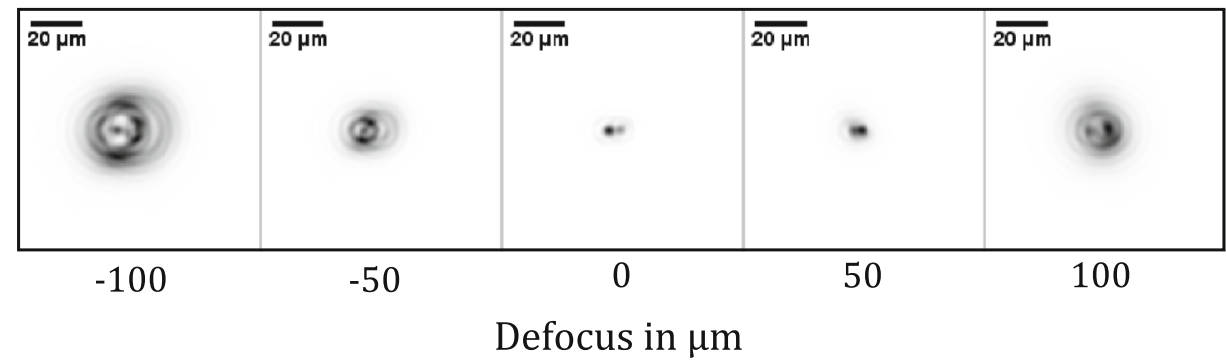

position can be determined. Typically less than one third spot diameter is used [8]. The angular sensitivity then depends on the beam diameter and wavelength of the beam $\varphi=\lambda / 3 D$. For the Petawatt beamline, this gives an angular sensitivity of $0.59 \mu \mathrm{rad}$. Residual dispersion seen in single pass after this alignment process points to inaccuracies in the collimation. Using the focal spot, scans can establish whether the beam is divergent or convergent and can be used to set the collimation of the beam. The nonsymmetrical collapse of the spots either side of focus is different in the two cases of $+R$ and $-R$ (Fig. 7). The point at which the centres of the spots overlap in all three cases is the focal plane of the parabola. On which side of the beam focus this occurs identifies convergence or divergence. This has been practically demonstrated on the Petawatt beamline (Fig. 8), which shows the dichroic laser through focus before correction that corresponds to a convergent beam with a wavefront radius of curvature of $35 \mathrm{~km}$. Applied correctly, this method will result in a radius of curvature set to the limit given by Eq.(1). Any remaining temporal effects on the pulse after this will be due to higher order aberrations within the beam.

\section{Conclusions}

We have presented analysis and modelling of the link between laser collimation and dispersive effects on CPA systems. These show that in a single pass compressor configuration an un-collimated beam can present itself through dispersion in the focal spot, which could be misinterpreted and attributed to dispersion from misaligned gratings leading to under performance of the laser system. The sensitivity of some traditional collimation techniques has been tested and shown not to have the accuracy required by single pass compressors of large beams. We have presented a technique for compressor alignment which can be used to collimate the beam to a higher degree than with standard methods.

Open Access This article is distributed under the terms of the Creative Commons Attribution License which permits any use, distribution, and reproduction in any medium, provided the original author(s) and the source are credited.

\section{References}

1. D. Strickland, G. Mourou, Opt. Commun. 56, 219 (1985)

2. C.N. Danson, P.A. Brummitt, R.J. Clarke, J.L. Collier, B. Fell, A.J. Frackiewicz, S. Hancock, S. Hawkes, C. Hernandez-Gomez, P. Holligan, M.H.R. Hutchinson, A. Kidd, W.J. Lester, I.O. Musgrave, D. Neely, D.R. Neville, P.A. Norreys, D.A. Pepler, C.J. Reason, W. Shaikh, T.B. Winstone, R.W.W. Wyatt, B.E. Wyborn, Nucl. Fusion 44, S239-S246 (2004)

3. P. Maine, D. Strickland, P. Bado, M. Pessot, G. Mourou, IEEE J. Quantum Electron. 24(2), 398 (1988)

4. O.E. Martinez, J. Opt. Soc. Am. 3(7), 929 (1986)

5. http://www.radiantzemax.com

6. G. Pretzler, A. Kasper, K.J. Witte, Appl. Phys. B 70, 1 (2000)

7. M.E. Riley, M.A. Gusinow, Appl. Opt. 16, 2753 (1977)

8. J.L. Collier, C. Hernandez-Gomez, S. J. Hawkes, J. Smith, T. B. Winstone, C. N. Danson, R. J. Clarke, D. Neely, Ch. Ziener, T. Strange, A. J. Frackiewicz, Central Laser Facility Annual Report 2002/2003, 168 (2003) 